\title{
Nucleosynthetic imprints from classical nova explosions: past to present
}

\author{
Jordi José ${ }^{1}$ \\ Dept. Física i Enginyeria Nuclear, EUETIB, Universitat Politècnica de Catalunya (UPC), \\ \& Institut d'Estudis Espacials de Catalunya (IEEC) \\ C. Urgell 187, E-08036 Barcelona, Spain \\ jordi.jose@upc.edu
}

\section{Margarita Hernanz}

Institut de Ciències de l'Espai (ICE/CSIC), \& Institut d'Estudis Espacials de Catalunya (IEEC)

Campus Universitat Autònoma de Barcelona, Facultat de Ciències, Torre C5 - parell, 2a planta,

E-08193 Bellaterra (Barcelona), Spain

hernanzeieec.uab.es

Classical novae are dramatic stellar explosions that take place in the accreted envelopes of white dwarfs in close binary systems. These unique cataclysmic events constitute a crucible where different scientific disciplines merge, including astrophysics, nuclear and atomic physics, cosmochemistry, high-energy physics or computer science. In this paper, we will focus on the nucleosynthesis accompanying such explosions. Theoretical predictions will be compared with the elemental abundances inferred from observations of the nova ejecta as well as with the isotopic abundance ratios measured in meteoritic grains. Special emphasis will be given to the interplay between nova outbursts and the Galactic abundance pattern. Results from recent simulations of nova explosions in cataclysmic primordial binaries will be also outlined. Finally, we will stress the key role played by nuclear physics in our understanding of these explosive phenomena by means of recent experiments and a thorough account of the impact of nuclear uncertainties. Hints on possible key nuclear physics experiments will be given.

10th Symposium on Nuclei in the Cosmos

Mackinac Island, Michigan, USA

27 July-1 August, 2008

\footnotetext{
1 Speaker
} 


\section{Introduction}

Classical novae outbursts have captivated the interest of astronomers for more than two millennia. They display a spectacular and fast rise in optical luminosity, reaching peak values of $\sim 10^{4}-10^{5} \mathrm{~L}_{\odot}$. Indeed, the closest novae are visible to the naked eye.

Observational analysis of old novae by Walker and Kraft [18,27] helped to settle their binary nature: these systems consist of a large, cool main sequence star that fills its Roche lobe, and a small white dwarf star. The two stellar components are so close $\left(\mathrm{P}_{\text {orb }}\right.$ $\sim 1-10 \mathrm{hr}$ ) that mass transfer episodes through the inner lagrangian point of the system ensue. The material carries angular momentum, and hence, it is not directly transferred onto the surface of the white dwarf star. Instead, it forms an accretion disk. Ultimately, a fraction of this H-rich material spirals in and ends up on top of the white dwarf, where it accumulates in semidegenerate conditions, paving the road for a thermonuclear runaway (hereafter, TNR).

Several hydrodynamic computations of nova outbursts have described in detail the expected chemical abundance pattern in the ejecta $[11,17,26$, and references therein]. Due to the high temperatures achieved in the envelope during the TNR, with typical peak values reaching $\sim(2-3) \times 10^{8} \mathrm{~K}$, novae are expected to eject significant amounts of nuclear processed material into the interstellar medium, in non-solar proportions. This has raised the issue of the potential role of classical novae in the Galactic abundances. Typically, $10^{-4}-10^{-5} \mathrm{M}_{\odot}$ are ejected per nova outburst, contributing to the enrichment of the interstellar medium in a handful of nuclear species, mainly ${ }^{13} \mathrm{C}$, ${ }^{15} \mathrm{~N},{ }^{17} \mathrm{O}$, and to some extent, ${ }^{7} \mathrm{Li}\left(\mathrm{CO}\right.$ novae) and ${ }^{26} \mathrm{Al}$ (ONe novae).

This so-called thermonuclear runaway model for the nova outburst (see $[13,15]$, for recent reviews) accounts fairly well for the gross observational properties that define these events, including the shape of the light curve, the maximum brightness (or luminosity) attained, and the chemical composition of the ejected shells.

It is important to recall that the nuclear history of the explosion is somewhat imprinted in the final chemical abundance pattern, revealing the characteristic timescale of the TNR as well as the temperatures (and hence, the nuclear processes) to which the envelope material has been exposed. It is also worth noting that despite of the problems associated with the modeling of such explosions (mainly, the nature of the mixing mechanism that operates at the core-envelope interface, as well as the overall amount of material that is ejected [16]), there is, in general, good agreement between theory and observations as regards nucleosynthesis. This includes the inferred atomic abundances and a plausible nucleosynthetic endpoint around $\mathrm{Ca}[11,17,26]$.

\section{The nova nuclear symphony}

From the nuclear physics viewpoint, novae are unique stellar explosions: their limited nuclear activity, which involves about a hundred relevant species $(\mathrm{A}<40)$ linked through a (few) hundred nuclear reactions, as well as the limited range of temperatures achieved in such explosions $(10-400 \mathrm{MK})$, allow us to rely primarily on experimental information [15]. 
Contrary to other (related) astrophysical explosive sites, during classical nova outbursts the main nuclear path runs close to the valley of stability, and is driven by $(\mathrm{p}, \gamma),(\mathrm{p}, \alpha)$ and $\beta^{+}$reactions. Hence, the contribution from any $(\mathrm{n}, \gamma)$ or $(\alpha, \gamma)$ reaction (including ${ }^{15} \mathrm{O}(\alpha, \gamma)$ ) becomes negligible. Different studies have focused on the role played by nuclear uncertainties on the overall nucleosynthetic pattern accompanying nova explosions [9]. Recent improvements have been achieved to this respect. Actually, the list of reactions whose uncertainty has a strong impact on the final yields has been dramatically reduced (see contributions from Adekola, Banu, Beer, Chen, Chipps, Dalouzy, Deibel, Moazen, Peplowski, Pittman, Ruiz, Sergi, Trache, Wiedenhoever, and Wrede, in this Conference). The main interest is focused now on the challenging reactions ${ }^{18} \mathrm{~F}(\mathrm{p}, \alpha){ }^{15} \mathrm{O},{ }^{25} \mathrm{Al}(\mathrm{p}, \gamma){ }^{26} \mathrm{Si},{ }^{30} \mathrm{P}(\mathrm{p}, \gamma){ }^{31} \mathrm{~S}$.

\section{Novae and presolar grains}

Infrared observations have revealed that classical novae often form dust (and grains) in their expanding shells ejected into the interstellar medium as a consequence of violent outbursts $[5,6]$. A fraction of this dust forms aggregates that assemble as meteorites. Recent experimental efforts have led to the identification of presolar grains from primitive meteorites (such as the Acfer 094 or Murchison), characterized by huge isotopic anomalies that can be linked to a suite of nucleosynthetic processes that took place in their parent stellar sources. So far, silicon carbide (SiC), graphite $(\mathrm{C})$, diamond (C), silicon nitride $\left(\mathrm{Si}_{3} \mathrm{~N}_{4}\right)$, silicates, and oxides, such as corundum $\left(\mathrm{Al}_{2} \mathrm{O}_{3}\right)$ or spinel $\left(\mathrm{MgAl}_{2} \mathrm{O}_{4}\right)$, have been identified as presolar grains (see [4,19,20,21,28], for recent reviews). Ion microprobe analyses of individual grains have indeed revealed a variety of isotopic signatures that point toward several stellar sources, such as asymptotic giant branch stars (AGBs) or supernovae (SNe).

The question is: are there any presolar nova grains among the different grain populations identified so far? Since the first studies of dust formation in classical novae by Clayton and Hoyle [3], all efforts devoted to the identification of potential nova grains relied mainly on the search for low ${ }^{20} \mathrm{Ne} /{ }^{22} \mathrm{Ne}$ isotopic ratios (with ${ }^{22} \mathrm{Ne}$ attributed to ${ }^{22} \mathrm{Na}$ decay, a signature of a classical nova). Indeed, these authors pointed out several isotopic signatures that may help in the identification of nova candidate grains, based in large overproductions of ${ }^{13} \mathrm{C},{ }^{14} \mathrm{C},{ }^{18} \mathrm{O},{ }^{22} \mathrm{Na},{ }^{26} \mathrm{Al}$, or ${ }^{30} \mathrm{Si}$. Twenty-five years later, our current understanding of the mechanism of nova explosions make ourselves confident on most of these predictions [14], except for ${ }^{14} \mathrm{C}$, which lays beyond the main nuclear path for novae, and ${ }^{18} \mathrm{O}$ (slightly overproduced in novae, but its relevance fades in front of the huge overabundances expected for ${ }^{17} \mathrm{O}$ ).

In fact, a rare variety of $\mathrm{SiC}$ grains $(<1 \%)$, together with a couple of graphite grains, that exhibit a suite of isotopic signatures characteristic of classical nova outbursts, have been reported in the recent years $[1,2]$. 


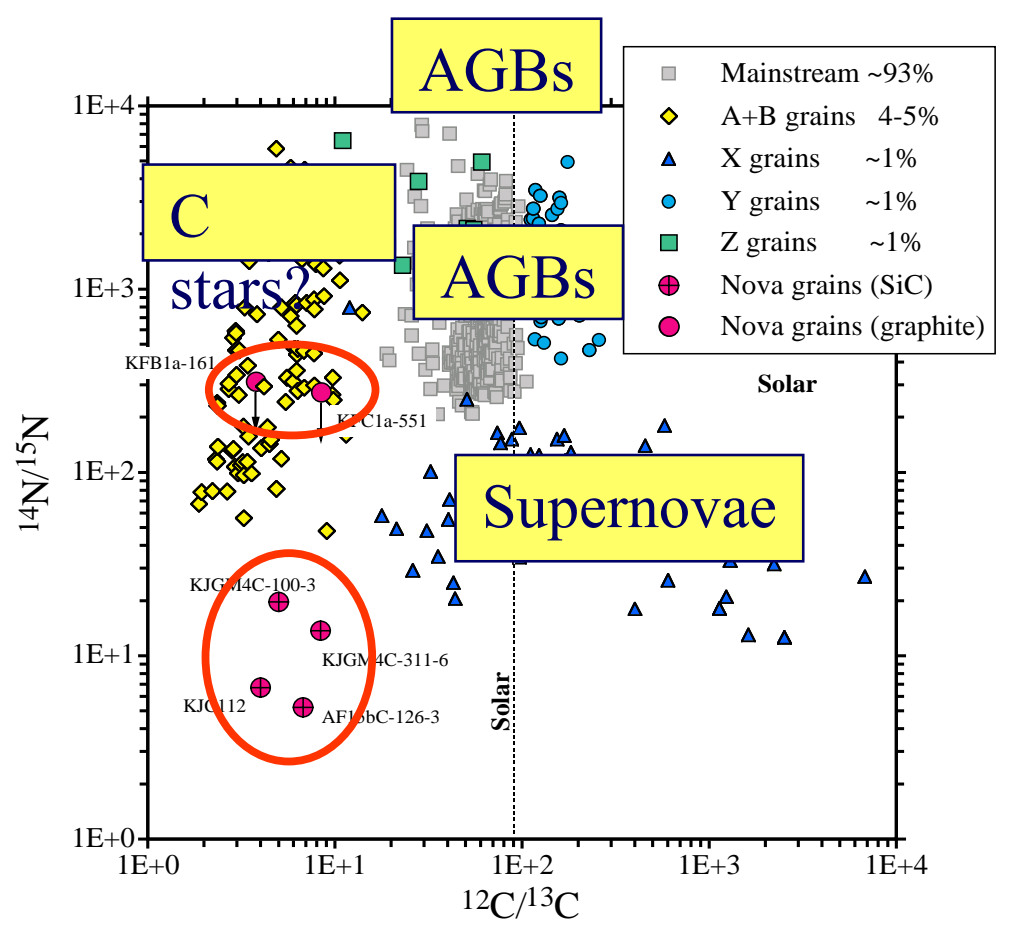

Fig. 1: Carbon versus nitrogen isotopic ratios for $\mathrm{SiC}$ grains (adapted from Amari et al. [1]).

This set of grains was identified on the basis of a handful of different isotopic ratios, simultaneously determined: low ${ }^{12} \mathrm{C} /{ }^{13} \mathrm{C}$ and ${ }^{14} \mathrm{~N} /{ }^{15} \mathrm{~N}$ ratios, high ${ }^{30} \mathrm{Si} /{ }^{28} \mathrm{Si}$, and close-to-solar ${ }^{29} \mathrm{Si} /{ }^{28} \mathrm{Si} ;{ }^{26} \mathrm{Al} /{ }^{27} \mathrm{Al}$ and ${ }^{22} \mathrm{Ne} /{ }^{20} \mathrm{Ne}$ ratios have been determined for some of these grains, with values compatible with model predictions.

But concerns about the likely nova paternity of these grains have recently been raised [24] on the basis of new measurements performed on three additional micronsized $\mathrm{SiC}$ grains isolated from the Murchison meteorite: these grains have very low ${ }^{12} \mathrm{C} /{ }^{13} \mathrm{C}$ and ${ }^{14} \mathrm{~N} /{ }^{15} \mathrm{~N}$ ratios, similar to the ones reported for the nova candidate grains $[1,2]$, but a number of additional imprints (mainly non-solar Ti features) suggesting instead a supernova origin.

It must be emphasized, however, that there is yet a scarce number of nova candidate grains. Hence, conclusions cannot be statistically sound. Moreover, it is not clear if one can claim the presence of Ti features in the Amari et al. samples (hereafter, A01) based on measurements performed in the Nittler and Hoppe sample (hereafter, NH05). After all, the isotopic signatures of both samples are not identical: grains from the NH05 sample have much larger ${ }^{26} \mathrm{Al} /{ }^{27} \mathrm{Al}$ ratios and are more depleted in ${ }^{29} \mathrm{Si}$ than grains from the A01 sample. Furthermore, grain M11-334-2 is defficient in ${ }^{30} \mathrm{Si}$ with respect to solar (whereas ${ }^{30} \mathrm{Si}$ excesses are expected in the ejecta from ONe novae). In summary, it is not clear if both samples correspond to the same progenitor. Clearly, we need to rely on a wider range of isotopic determinations for a proper identification of the stellar source.

Other nova candidate grains have been proposed: indeed, the $\mathrm{SiC}$ grain $240-1$, also isolated from the Murchison meteorite [23], exhibits both ${ }^{12} \mathrm{C} /{ }^{13} \mathrm{C}$ and ${ }^{14} \mathrm{~N} /{ }^{15} \mathrm{~N}$ ratios lower than for any other reported presolar grain. These isotopic features are 
consistent with pure nova ejecta with white dwarf masses of $\sim 1.0-1.2 \mathrm{M}_{\odot}$. A supernova origin is unlikely because of such low ${ }^{12} \mathrm{C} /{ }^{13} \mathrm{C}$ ratios. However, the ${ }^{29} \mathrm{Si}$ excesses measured in this grain do not match the usual predictions from models (which suggest ${ }^{29} \mathrm{Si}$ deficits, with respect to solar, instead).

Also a putative nova origin has been attributed to the oxide grain T54 [22], with ${ }^{16} \mathrm{O} /{ }^{17} \mathrm{O} \sim 71$ and ${ }^{16} \mathrm{O} /{ }^{18} \mathrm{O} \sim 2000$, suggesting its likely condensation in the nova shells ejected from an outburst on a $0.8 \mathrm{M}_{\odot} \mathrm{CO}$ white dwarf. Unfortunately, no additional isotopic determinations were made on this grain.

\subsection{Ti synthesis in novae}

In this section we want to address a very important point: does the presence of non-solar Ti ratios point towards a supernova origin or can novae, under some conditions, account for these specific signatures? In fact, titanium is very close to the classical nucleosynthetic endpoint for nova explosions: calcium. Therefore, we only need sligthly more violent outbursts to extend somewhat the nuclear activity up to Ti.

There are two basic ways to achieve that:

* Explosions in lower luminosity (colder) white dwarfs, or in low-accretion rate episodes [12].

* Explosions in metal-defficient envelopes, such as those expected in primordial nova systems [10].
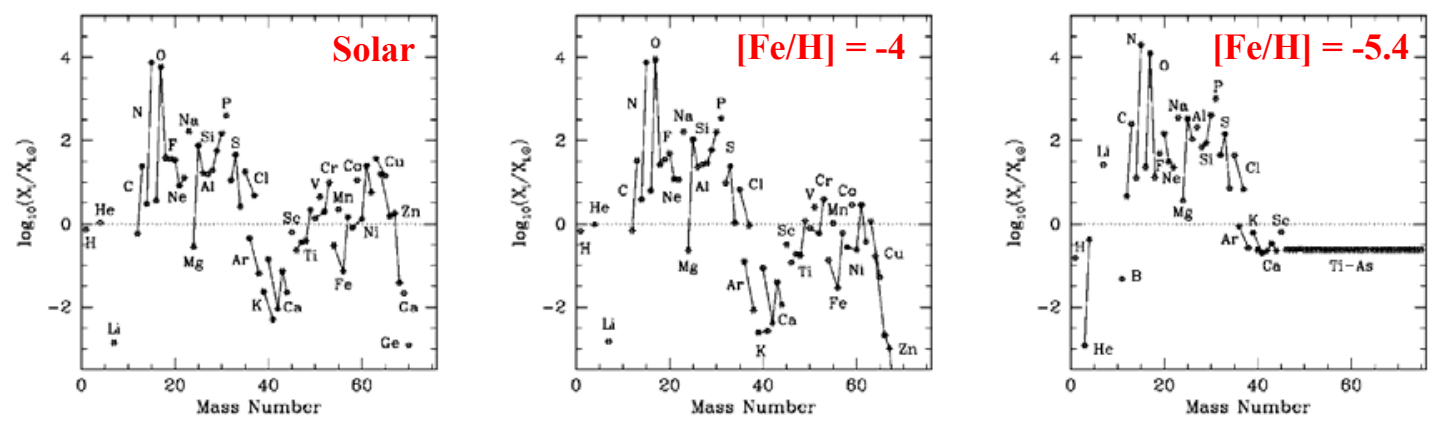

Fig. 2. Mean chemical composition in the ejecta, with respect to solar, as a function of the envelope metallicity (adapted from José et al. [10]).

Primordial novae designate nova-like stellar explosions that take place in the accreted envelopes on top of white dwarfs in cataclysmic primordial binaries $\left(\mathrm{Z}_{\mathrm{zams}} \sim\right.$ $0)$. It is likely that in these systems, the stellar companion that transfers material onto the white dwarf could be very metal-poor. This may have dramatic consequences for the final nova explosion. To test this, we have performed several hydrodynamic simulations of primordial novae, parameterizing the metallicity in the accreted material. In contrast with previous studies [11,26], these models have been computed without preenrichment; mixing is assumed to take place later in the explosion, according to detailed multidimensional studies by Glasner et al. [7,8]: the build-up of convective eddies at the envelope's base causes shear flow at the core/envelope interface (Kelvin-Helmholtz 
instabilities); hence, pure "solar-like" accreted material can be enriched at the late stages of the TNR by some sort of convective overshoot, leading to a powerful nova!

Glasner's results are used to determine when and how fast material is mixed at the core-envelope interface in our 1-D hydrodynamic simulations. From the nucleosynthetic viewpoint, the interest of this study is illustrated in Fig. 2: an extension of the main nuclear path towards heavier species follows the decrease of the metallicity in the stellar companion. In summary, non-solar Ti features can be obtained for those primordial novae (also for those computed with slightly lower luminosities or lower mass-accretion rates). Hence, Ti does not necessarily rule out a possible nova paternity (except for ${ }^{44} \mathrm{Ti}$ excesses, clearly pointing towards a supernova origin).

\subsection{Discussion}

The possible link between classical nova outbursts and the presolar grain populations raised some questions and comments during this Conference. First, it should be emphasized that, according to the predicted abundances in the ejecta, nova may contribute to the oxide grain population; furthermore, their isotopic signatures suggest that some nova grains can be embedded in the $\mathrm{A}+\mathrm{B}$ subclass of $\mathrm{SiC}$ grains (at least, for those grains characterized by low ${ }^{12} \mathrm{C} /{ }^{13} \mathrm{C}$ ratios that do not show large ${ }^{29} \mathrm{Si}$ excesses). Concerning the possible search for other nova signatures in presolar grains, such as $\mathrm{S}$ excesses, it must be stressed that $\mathrm{S}$ isotopes may be affected by contamination of the samples -due to the use of sulfuric acid during the separation process-, and it is not clear if $\mathrm{S}$ can eventually condense into $\mathrm{SiC}$ grains. In turn, $\mathrm{P}$ has only one stable isotope which makes the analysis very difficult.

\section{Reactions triggering nova outbursts}

Classically, nova explosions are thought to be triggered by ${ }^{12} \mathrm{C}(\mathrm{p}, \gamma)$. Hence, ${ }^{12} \mathrm{C}$ constitutes the single, most important isotope to determine the strength of the explosion. But a recent study [25] pointed out that this may not be true at lower metallicities. It has to be stressed though, that this study explored only extremely high mass accretion rates (in the range $\left.10^{-9}-3 \times 10^{-7} \mathrm{M}_{\odot} \cdot \mathrm{yr}^{-1}\right)$, and a very narrow range of metallicities $(\mathrm{Z}=0.1-$ $\left.5 \mathrm{Z}_{\odot}\right)$. The authors concluded that convection sets in powered by ${ }^{14} \mathrm{~N}(\mathrm{p}, \gamma)$, following a quick ${ }^{12} \mathrm{C}$-depletion by ${ }^{12} \mathrm{C}(\mathrm{p}, \gamma)$.

Here, we will briefly discuss which reaction(s) drives the TNR in lowmetallicity novae. In particular, we will analyze two cases $\left(\mathrm{Z}=\mathrm{Z}_{\odot} / 100\right.$ [Model B], and $\mathrm{Z}_{\odot} / 250,000$ [Model $C$ ], the latter corresponding to one of our primordial nova systems), in the context of explosions onto a $1.35 \mathrm{M}_{\odot}$, ONe white dwarf, accreting at a rate of $2 \times 10^{-10} \mathrm{M}_{\odot} \cdot \mathrm{yr}^{-1}$.

Snapshots of the evolution of Model B $\left(Z=Z_{\odot} / 100\right)$ are shown in Fig. 3: early in the course of the explosion (upper panel), the main nuclear activity is driven by the $p$ $p$ chains (because of the lack of CNO catalysts). 

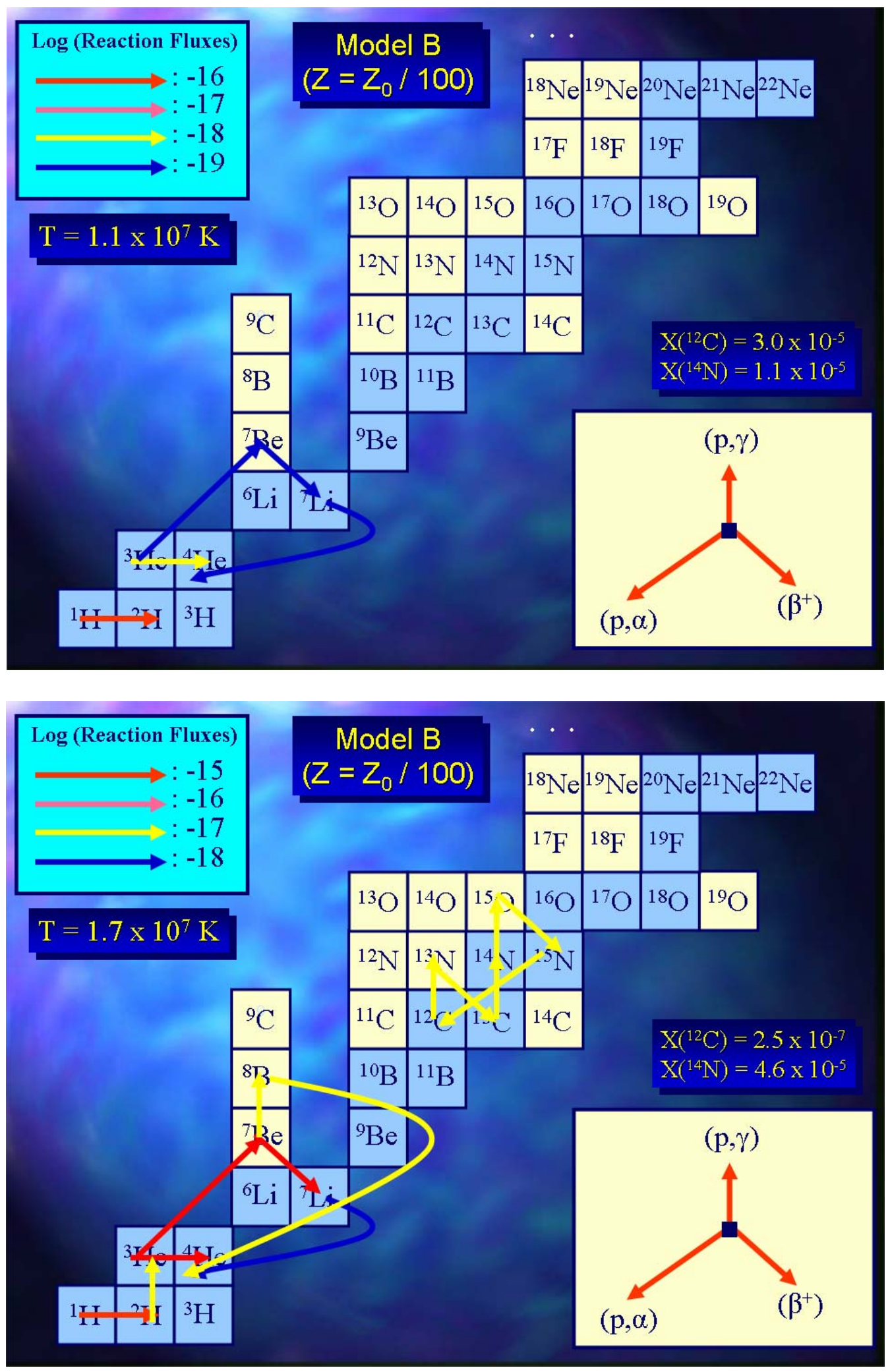


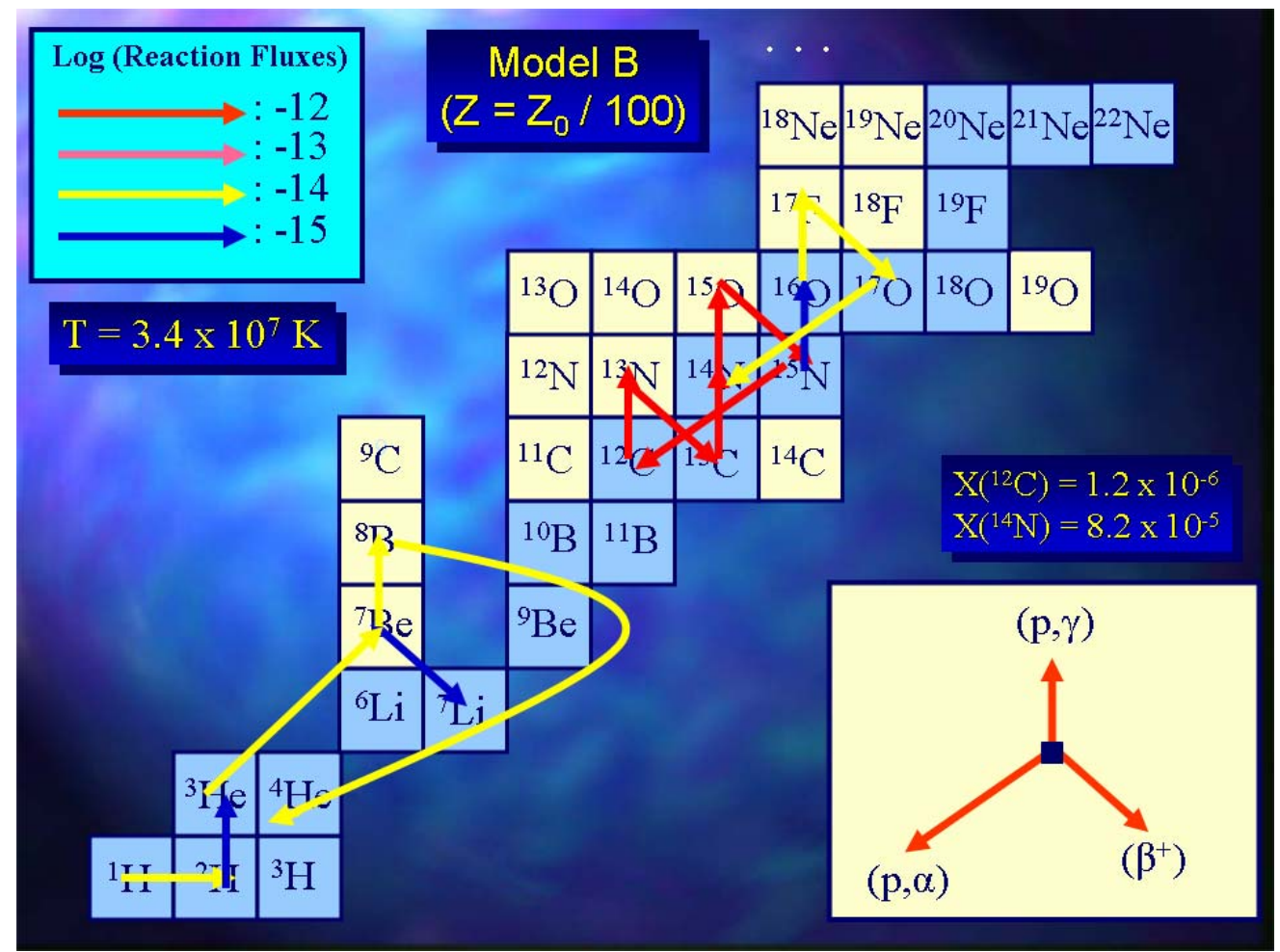

Fig. 3: Snapshots of the nuclear activity at the base of a nova envelope with $Z=Z_{\odot} / 100$ (see text, for details). Arrows represent reaction fluxes (number of reactions per unit time and volume).

As discussed by Shen \& Bildsten [25], we report a significant depletion of ${ }^{12} \mathrm{C}$ by two orders of magnitude, which translates into a rise in the ${ }^{14} \mathrm{~N}$ abundance (middle panel). However, reactions fluxes for ${ }^{12} \mathrm{C}(\mathrm{p}, \gamma)$ and ${ }^{14} \mathrm{~N}(\mathrm{p}, \gamma)$ are still very similar, because of efficient recycling into ${ }^{12} \mathrm{C}$ by ${ }_{14}^{15} \mathrm{~N}(\mathrm{p}, \alpha)$.

Certainly, the importance of ${ }^{14} \mathrm{~N}(\mathrm{p}, \gamma)$ is unambiguosly established, but contribution from ${ }^{12} \mathrm{C}(\mathrm{p}, \gamma)$ (as well as from additional reactions of the $\mathrm{CNO}$ cycle) cannot be ruled out. The same applies at slightly more advanced stages, just at the time when convection sets in (lower panel).

A similar behavior is found at much lower metallicities of Model C $\left(\mathrm{Z}_{\odot} / 250,000\right.$; Fig. 4), somewhat representative of primordial novae.

A more extensive and detailed analysis of the reactions triggering nova outbursts at different conditions, from a hydrodynamic framework, is currently underway.

\section{Acknowledgments}

This research has been funded by the spanish MEC grants AYA2007-66256 and ESP200761593, by the catalan AGAUR grant 2005-SGR00378 and by FEDER funds. 


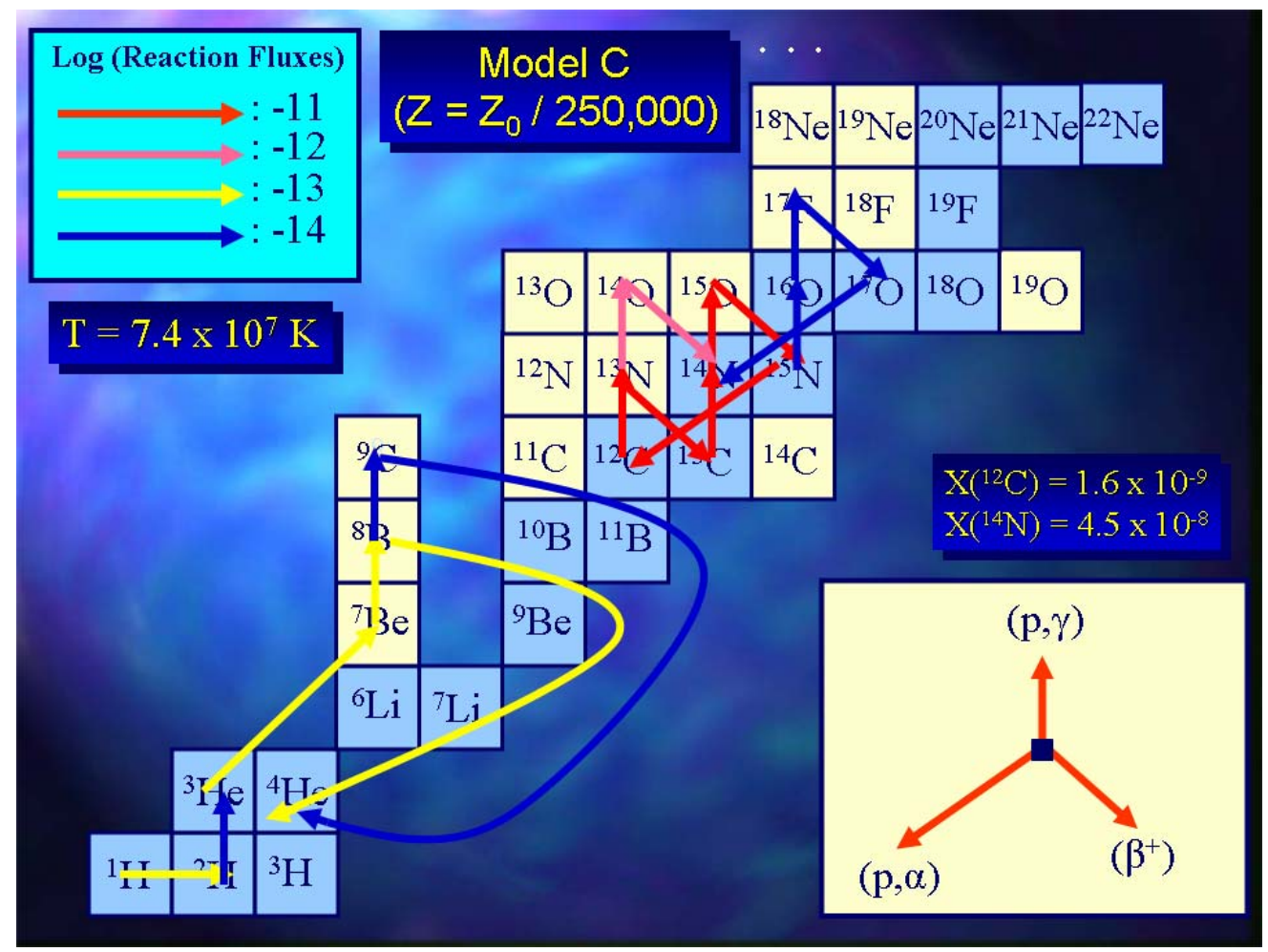

Fig. 4: Main nuclear activity at the base of a nova envelope with $Z=Z_{\odot} / 250,000$ (see text, for details), around the time when convection sets in.

\section{References}

[1] S. Amari, Presolar grains from novae: their isotopic ratios and radioactivities, New Astr. Rev. 46 (2002) 519 .

[2] S. Amari, X. Gao, L. Nittler, E. Zinner, J. José, M. Hernanz, \& R. Lewis, Presolar grains from novae, ApJ 551 (2001) 1065.

[3] D.D. Clayton, \& F. Hoyle, Grains of anomalous isotopic composition from novae. ApJ 203 (1976) 490.

[4] D.D. Clayton, \& L.R. Nittler, Astrophysics with presolar stardust. ARA\&A 42 (2004) 39.

[5] R.D. Gehrz, Infrared and radio observations of classical novae: physical parameters and abundances in the ejecta, in Proc. Int. Conf. on Classical Nova Explosions, M. Hernanz and J. José (eds.), AIP: New York (2002) 198.

[6] R.D. Gehrz, J.W. Truran, R.E. Williams, \& S.M. Starrfield, Nucleosynthesis in classical novae and its contribution to the interstellar medium, PASP 110 (1998) 3.

[7] S.A. Glasner, \& E. Livne, Convective hydrogen burning down a nova outburst, ApJ 445 (1995) L149.

[8] S.A. Glasner, E. Livne, \& J.W. Truran, Reactive flow in nova outbursts, ApJ 475 (1997) 754. 
[9] C. Iliadis, A. Champagne, J. José, S. Starrfield, \& P. Tupper, The effects of thermonuclear reactionrate variations on nova nucleosynthesis: a sensitivity study, ApJS 142 (2002), 105.

[10] J. José, E. García-Berro, M. Hernanz, \& P. Gil-Pons, The First Nova Explosions, ApJ 662 (2007) L103.

[11] J. José, \& M. Hernanz, Nucleosynthesis in classical novae: CO vs. ONe white dwarfs, ApJ 494 (1998) 680.

[12] J. José, \& M. Hernanz, The origin of presolar nova grains, Meteorit. Planet. Sci. 42 (2007) 1135

[13] J. José, \& M. Hernanz, Nucleosynthesis in classical nova explosions, JPG 34 (2008) R431.

[14] J. José, M. Hernanz, S. Amari, K. Lodders, \& E. Zinner, The imprint of nova nucleosynthesis in presolar grains, ApJ 612 (2004) 414.

[15] J. José, M. Hernanz, \& C. Iliadis, Nucleosynthesis in classical novae, NPA 777 (2006) 550.

[16] J. José, \& S. Shore, Observational Mysteries and Theoretical Challenges for Abundance Studies, in Classical Novae, M. Bode and A. Evans (eds.), Cambridge University Press, Cambridge (2008) 121.

[17] A. Kovetz, \& D. Prianik, The composition of nova ejecta from multicycle evolution models, ApJ 477 (1997) 356.

[18] R.P. Kraft, in Advances in astronomy and astrophysics II,, New York: Academic Press, (1963) 43.

[19] K. Lodders, \& S. Amari, Presolar grains from meteorites: remnants from the early times of the solar system, Chemie der Erde 65 (2005) 93.

[20] M. Lugaro, Stardust from meteorites: an introduction to presolar grain, Singapore: World Scientific Publ. (2005).

[21] B.S. Meyer, \& E.K. Zinner, in Meteorites and the early solar system II, Tucson: Univ. Arizona Press, (2006), 69.

[22] L.R. Nittler, Presolar Oxide Grains in Meteorites, in Proceedings, Astrophysical Implications of the Laboratory Study of Presolar Materials (1997), 59.

[23] L.R. Nittler, C.M.O.'D. Alexander, \& A.N. Nguyen, Extreme ${ }^{13} C$ and ${ }^{15} N$ enrichments in a Murchison presolar SiC grain, Abst. \#5316, Meteoritic Soc. Meeting (2006).

[24] L. R. Nittler, \& P. Hoppe, Are presolar silicion carbide grains from novae actually from supernovae? ApJ 631 (2005) L89.

[25] K. J. Shen, \& L. Bildsten, The effect of composition on nova ignitions, ApJ (submitted, 2008) [arXiv:0805.2160v1]

[26] S. Starrfield, J.W. Truran, M.C. Wiescher, \& W.M. Sparks, Evolutionary sequences for Nova V1974 Cygni using new nuclear reaction rates and opacities, MNRAS 296 (1998) 5022.

[27] M.F. Walker, Nova DQ Herculis (1934): an eclipsing binary with very short period, PASP 66 (1954) 230.

[28] E.K. Zinner, Meteorites, comets, and planets, in Treatise on geochemistry I. Oxford: Elsevier, (2004) 17. 